\title{
Creating Communications, Computing, and Networking Technology Development Road Maps for Future NASA Human and Robotic Missions
}

\author{
Kul Bhasin ${ }^{1}$, Jeffrey L. Hayden ${ }^{2}$ \\ ${ }^{I}$ NASA Glenn Research Center, 21000 Brook Park Rd., Cleveland, OH 44135, USA \\ ${ }^{2}$ Glenn Research Center/Infinite Global Infrastructures, L.L.C., West Chicago, IL 60185, USA \\ '216-433-3676,Fax:216-433-5050,Kul.B.Bhasin@nasa.gov
}

\begin{abstract}
For human and robotic exploration missions envisioned in the Vision for Exploration, roadmaps are needed for capability development and investments based on advanced technology developments. A roadmap development process was undertaken for the needed communications, computing, and networking capabilities and technologies for the future human and robotics missions. The underlying processes are derived from work carried out during development of the future space communications architecture, and NASA's Space Architect Office (SAO) defined formats and structures for accumulating data. Interrelationships were established among emerging requirements, the capability analysis and technology status, and performance data. After developing an architectural communications and networking framework structured around the assumed needs for human and robotic exploration, in the vicinity of Earth, Moon, along the path to Mars, and in the vicinity of Mars, information was gathered from expert participants. This information was used to identify the capabilities expected from the new infrastructure and the technological gaps in the way of obtaining them. We define realistic, long-term space communication architectures based on emerging needs and translate the needs into interfaces, functions, and computer processing that will be required. In developing our roadmapping process, we defined requirements for achieving end-to-end activities that will be carried out by future NASA human and robotic missions. This paper describes: 1) the architectural framework developed for analysis; 2) our approach to gathering and analyzing data from NASA, industry, and academia; 3) an outline of the technology research to be done, including milestones for technology research and demonstrations with timelines; and 4) the technology roadmaps themselves.
\end{abstract}

\section{INTRODUCTION}

In January 2004, NASA embarked on a new quest for the human and robotic exploration of the solar systems. This vision (NASA, 2004a) has already transformed the Agency and added human and robotic exploration as a major component of its mission focus. Furthermore, the vision requires innovative technologies and infrastructure to be deployed in support of the exploration missions. Achieving this vision within the available budget requires realistic, well-planned roadmaps. The process that was used in creating the roadmaps has evolved and improved dramatically as new tools and methods of analysis have become available. The objective of this work was the formulation of the development roadmaps for the space communications, networking and processing technologies needed for human and robotic exploration. Roadmaps for developing these technologies are needed because the capabilities of today's communications infrastructure cannot support the multipoint, bidirectional, and high-rate, end-to-end requirements for human exploration at distant places; nor does any capability exist to handle communications among multiple humans, robots, and science instruments on the surface of the Moon or Mars simultaneously as will be needed to support construction and exploration in an airless environment. The purpose of this paper is to describe the roadmapping process and how it was based on the multiple points of view-architectural, mission, and system of systems-to ensure that the roadmap thoroughly covers the communication, networking and computing capability and technology needs.

The timeline provided in the exploration vision served as a template for developing the technology roadmaps. The

This is a preprint or reprint of a paper intended for presentation at a conference. Because changes may be made before formal publication, this is made available with the understanding that it will not be cited or reproduced without the permission of the author. 

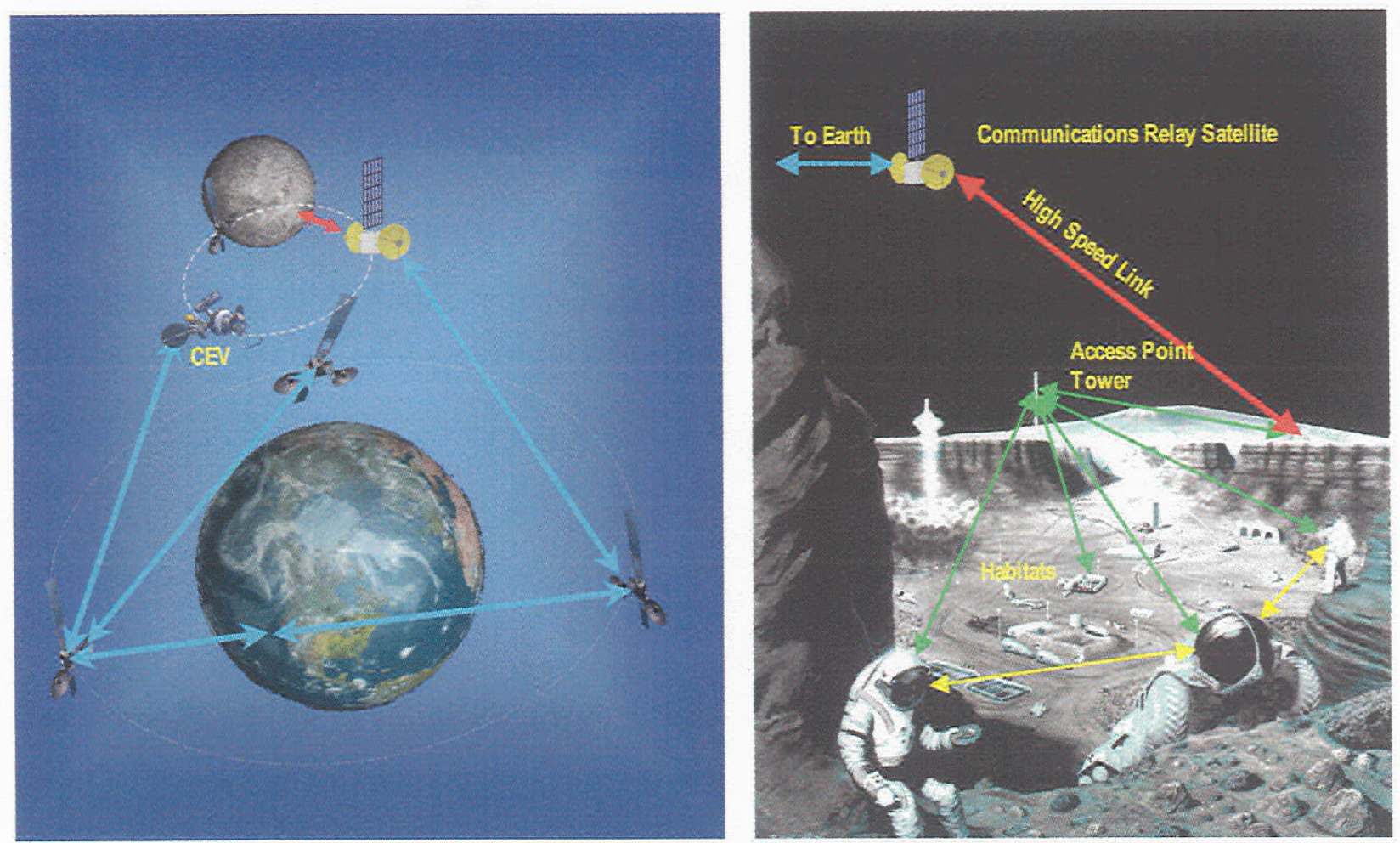

FIGURE 1. The Communications and Networking Architecture for Human and Robotic Exploration of the Moon Includes a High Data Rate Space Backbone Network Between the Earth and the Moon, the CEV and Communication Relay Satellites in Lunar Orbit, and Surface Wireless Local Area Networks to Provide End-to-End Communications Between Any Nodes.

Human and Robotic Exploration (H\&REx) of Moon and Mars is planned to begin after 2008 with the launch of a Crew Exploration Vehicle (CEV) that will undergo assembly in low Earth orbit. During the next phase, which starts around 2016, the CEV and crew will transport to the lunar vicinity after being fitted with a lunar landing system. The CEV may enter a halo orbit (FIGURE 1 around the gravitationally balanced Earth-Moon Lagrange point $\left(E M L_{1}\right)$ and serve as a way station for humans going to and from the lunar surface. The lunar surface missions will provide training for eventual human missions to Mars that may begin in the third phase, likely after 2030 . The same $\mathrm{CEV}$ location at $\mathrm{EML}_{1}$ is being considered as a possible waypoint for the Mars mission. This paper describes the process used in generating the roadmap for developing the technologies that will enable the capabilities that a modern communications, networking and processing infrastructure must provide to support these new missions.

First, we present the roadmap development process. Next, we describe the three points of view from which the roadmap information was gathered-architecture, mission, and system of systems perspectives. The capability breakdown structure, followed by the capability and technology data gathering and format are then described. Finally, the major technology gaps are identified and the technology development roadmaps are presented.

\section{ROADMAP DEVELOPMENT PROCESS}

The development process described here resulted in several possible technological solutions for providing a new infrastructure capable of autonomous routing communications between any pair of end point nodes on the network. This paper does not presume to provide the complete answer for in-space communications, networking and processing, but it does serve up several potential solutions. The purpose of this process is to provide a capability and technology framework that can be used as a guide in selecting new technologies when the aerospace and commercial communities propose them for development.

The process for gathering the capabilities and technologies needed for the Human and Robotic Exploration program is shown in FIGURE 2. The NASA Space Architect Office (SAO) identified the high-level requirements and capabilities for the space transportation infrastructure that will enable the H\&REx missions. The SAO also initiated 
the gathering of capabilities needed by all systems within the infrastructure during each mission phase of the H\&REx program. The needed communication capabilities were extracted from the general capabilities expressed in the space exploration vision. A capability breakdown structure (CBS) worksheet was initiated with the list of potential capabilities passed from the SAO. The CBS worksheet file was expanded into an Excel workbook to include technology data worksheets and a technology data summary worksheet. Mission and system experts at Goddard Space Flight Center (GSFC), Johnson Space Center (JSC), Jet Propulsion Lab (JPL), and Glenn Research Center (GRC) assisted in reviewing the CBS. Technology experts from GSFC, JPL, and GRC contributed to and reviewed the technology datasheets.

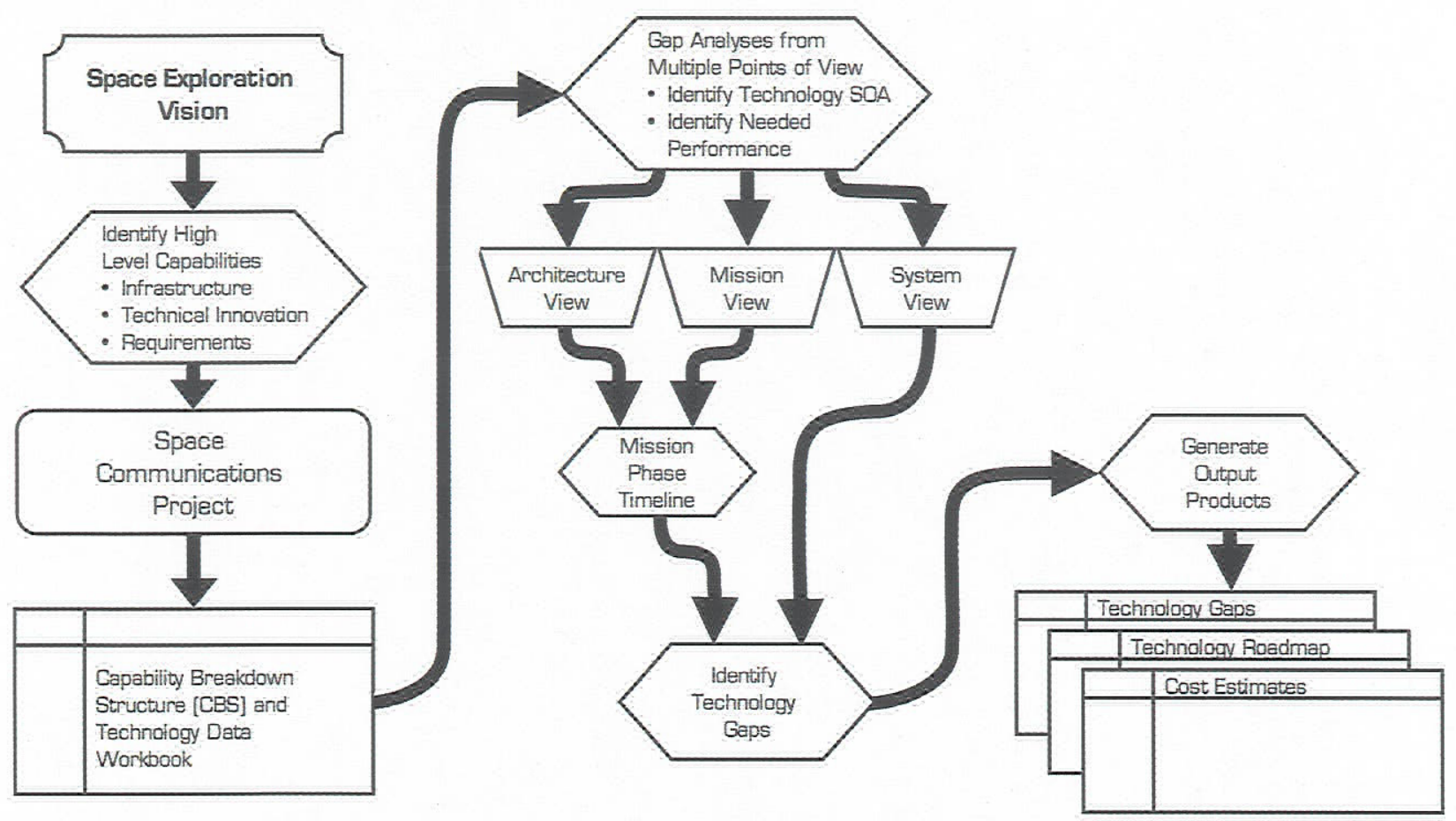

FIGURE 2. The Process for Developing the Capability Breakdown Structure, Identifying the Needed Technologies and Their Requirements, and the Roadmap for Developing the Technologies.

After capturing the capabilities needed in the space communications, networking and processing infrastructure to support the H\&REx missions and relevant technology inputs, the data was analyzed from the architectural, mission, and system of systems perspectives to determine the gaps in technology. The general mission and technology development timelines were determined by the SAO. The architectural and mission points of view were applied to determine when particular communication, networking and processing capabilities and technologies were needed to support the three main phases of the H\&REx missions. Technologies that will provide the capabilities needed to support the CEV assembly flights in Earth orbit by 2014 must reach Technology Readiness Level (TRL) (NASA, 1999) 6 by 2008. To support human missions to the Moon by 2020, the technologies have to be at TRL 6 by 2014. Human missions to Mars may begin after 2030, so those technologies must be at TRL 6 by 2023. The technology gaps were determined by comparing the needed performance against the state of art (SOA) performance of existing technology. If the SOA of an existing technology could provide a needed performance, that capability thread was declared mature and no further development was deemed necessary. A gap was identified when existing technology could not meet the performance needed by that technology. The results of the analyses were summarized in three ways: in a table of technology gaps, in a roadmap that identifies developing technologies to fill the gaps, and in yearly cost estimates for space communication, networking and processing technology development.

\section{Architectural Point of View}

The architectural view was based on prior work (Bhasin, 2004a; Bhasin, 2004b; Bhasin, 2002) that defined the capabilities needed for architecture elements: the space backbone that is needed to pipe large volumes of data 
bidirectionally among Earth, CEV, Moon, and Mars; the access networks that are needed to interface between the space backbones and the CEV or Moon or Mars; the proximity networks that include wireless surface local area networks for multipath, bidirectional, voice, video, and data communications among humans, robots, vehicles and habitats; and the vehicle local area networks needed to move data between subsystems and instruments on-board a spacecraft or inside a habitat. Technologies that address these architectural capabilities include: optical and microwave systems for bidirectional, space-to-space communications at greater than 1 gigabit per second (Gbps); surface terminal systems for interfacing to space backbone relay satellites or Earth ground stations at high data rates; on-board network routers that maintain addressed access between any nodes on the network; on-board and habitat servers to maintain local databases and provide domain name server services locally; architecture for a navigational network similar to GPS around Moon and/or Mars for surface navigation with a minimum number of satellites; miniature wearable wireless network modules, similar to 802.11 technologies, with processor-controlled directive antennas mounted on the astronaut's helmet; and, wireless local area network base stations for dispersal around areas of human and robotic surface activities to maintain communications and network connectivity to all nodes in the local area.

\section{Mission Point of View}

Capabilities needed from the mission point of view were obtained by distilling the communication component of the general capabilities described in design reference missions (DRM) that were similar in implementation to the H\&REx vision concept and from requirements gathered in previous years from the strategic plans of NASA enterprises. The SAO identified three design reference mission concepts as potential representatives of the mission phases of the H\&REx missions: the 2002 Orbital Aggregation \& Space Infrastructure Systems (OASIS) (Troutman, et. al., 2002) mission, the 2003 Architecture Study \#1 (NASA internal document), and the 1997 Mars Reference Mission with a 1998 Addendum (NASA, 1997-1999). The Architecture Study \#1 and OASIS concepts studied the assembly of large structures in space in low Earth orbit and in a halo orbit around the EML 1 point. Each had concepts similar to those for assembling CEV structures in Earth orbit and for operating the CEV in the vicinity of the Moon. Architecture Study \#1 included lunar landing scenarios and described mission concepts for transporting the CEV to Mars from EML $L_{1}$. The Mars Reference Mission and Addendum were developed prior to the other studies and utilized Saturn V-class rocket flights launched directly from Earth to Mars rather than being assembled in Earth orbit with pieces launched with smaller rockets. However, the Mars Reference Mission does discuss the more pertinent human and robotics mission on the surface of Mars.

During the initial phase of H\&REx missions, assembly of the CEV in space will need continuous, bidirectional, high data rate support from Earth orbiting relay satellites, such as the TDRSS or similar capability systems, to enable high definition video and robotic teleoperation coverage of assembly activities. Bidirectional voice, video, and data will be needed between the CEV, astronauts on Extra Vehicular Activities (EVAs), and the assembly robots in the CEV vicinity. Optical and microwave technologies for advancing the state of the art of space relays have been identified for supporting this early H\&REx phase.

The next phase of H\&REx missions brings humans in the CEV to the vicinity of the Moon, lands humans on the Moon, and supports human and robotic assembly of lunar surface structures and lunar surface exploration. Technologies that enable high data rate optical and microwave communication relay spacecraft in the lunar vicinity with on-board router networking will be pursued. These relay spacecraft must support communications from areas of the Moon that are not in the line of sight from Earth, such as the south pole or the far side of Moon. Technologies that enable high data rate optical and microwave surface terminals for communicating with the lunar relay spacecraft will be needed, including: inflatable antennas, Ka-band and 40 to $55 \mathrm{GHz}$ transceivers and laser communication systems, on-board and surface routers, and lunar surface servers. After humans land on the Moon, the wireless local area network has to support reliable bidirectional, multipoint communications and networking between tens of nodes. These surface networks must handle several voice, video, and data streams simultaneously during assembly and exploration activities. Technologies that will provide these capabilities are similar to the 802.11 and 802.16 systems that are well developed in the commercial world. NASA will adapt these technologies to its special needs by developing smart directive antennas on helmets and in vehicles, developing very light weight and portable cell tower-like relay pole technologies and systems, and modifying designs to withstand the lunar radiation environment and the extreme temperature excursions between lunar night and day. 
The Mars missions are similar to the lunar missions, as they should be. The Moon will be the practice area for the Mars missions. It is expected that technologies developed for the lunar missions will undergo spiral development to obtain higher all around capabilities than the lunar technologies. Longer range relay satellites will be needed, particularly to communicate around the sun when Mars and the humans on it are blocked from communicating with Earth during solar conjunction. The technologies will include those that enable optical and microwave communications at high data rates between the Earth, the in-space relays, and the Mars relays. Also included are the technologies needed for improved wireless surface networks. A few technologies were identified for development for this Mars phase, but it is likely that they will be revised as experience is gained with the lunar missions.

\section{System of Systems Point of View}

A system of systems approach to space communications is needed to address the strategic technical challenges described in the human and robotic technology (H\&RT) formulation plan (NASA, 2004b). A system of communications and network systems must be effective and flexible. It must also be a sustainable infrastructure; that is, it must be affordable, reliable and as safe as reasonably achievable in support of humans in space. The combination of modern networking technologies with standardized wireless communication interfaces is needed to provide an affordable, reliable, and flexible infrastructure that can expand to effectively meet the interactive requirements of each phase of the H\&REx mission. The continuous communications enabled by autonomously operated wireless network, processor and protocol technologies will provide the highest degree of safety for the human explorers. The system of systems that enables end-to-end communications between any pair of nodes on the space communications network consists of the integration of the space backbone network, the space wide area networks, the surface wireless local area networks, and the vehicle local area networks that were defined previously in the architectural point of view. Requirements from the system view were obtained from mission center presentation packages at the Space Communication Architecture Working Group, by direct solicitation from mission communication experts, from the Space Internet Workshops (NASA, 2000, 2002, 2003, 2004c), and from prior workshops on space communications and networking. Technologies were identified that standardize the hardware, software, and wireless interfaces, and that enable the disparate networks to intercommunicate. Technologies that enable intercommunication across networks include: software configurable radios capable of operating with various modulations and networking protocols over a wide frequency range; agile antennas capable of maintaining simultaneous links to multiple spatially dispersed nodes; and standardized media access control (MAC) layer protocols to enable access on demand to any network.

Technologies that provide autonomy in operations and in intercommunication between the networks were also identified for development. These technologies include: control processors for operating the communications equipment, the MAC layer protocols just mentioned; autonomous, in-space network management, configuration and processing control based on priority, traffic, and network resources; link optimization built into the antenna/transceiver/processor system to control beam focusing, transmitter power, data transmission rate, Doppler shift correction, and time delay correction to autonomously adapt to dynamically changing link conditions; and resource optimization software to preserve power while maximizing data transmission rates.

The system of systems concept includes navigation and relative position awareness. To enable nodes such as space relays, human and robotic spacecraft, or astronauts on EVAs to operate with maximum autonomy from Earth-based operations, these entities need to know where they are in-space, on a lunar or planetary surface, or relative to each other. Technologies that enable autonomous navigation and position knowledge include beacon functionality onboard communication relays that serve user spacecraft by providing guideposts for user navigation; low cost, low mass precision oscillators for keeping the correct time and protocols for synchronizing clocks between nodes; and GPS or radar-like technologies to provide relative position knowledge. Security in communications is also applied with secure protocols or encryption technologies at the wireless and wired interfaces in the integration of the systems.

\section{CAPABILITY INDENTIFICATION AND BREAKDOWN STRUCTURE}

FIGURE 3 shows the CBS down to level 3, with $4^{\text {th }}$ level bullet points for the four titles of the breakdown structure at level 3: 2.1.1 Space Backbone and Access, 2.1.2 Planetary Networks, 2.1.3 Security, and 2.1.4 Navigation. The $4^{\text {th }}$ 
level capabilities, addressed as bullets within each heading, are reasonably self-explanatory. In general, most of the communications and networking capabilities are captured under 2.1.1 and 2.1.2. The 2.1.3 Security capability is largely the application of typical functions such as authentication, intrusion detection, encryption, and decryption as implemented by software protocols and special hardware devices. 2.1.4 Navigation includes the hardware and software needed to implement beacon "lighthouse" references in space; capabilities to support ranging, velocity, and angle measurements to "known" relay spacecraft locations; low cost and mass precision oscillators; GPS-like capabilities with low satellite counts; and inter-spacecraft ranging and relative position measurement. A fifth title was added later because it was felt that the capability to support end-to-end communications between any nodes on the networks was not being clearly stated to the potential customers. 2.1.2.6 End-to-End was then added because the number 2.1.5 was taken. Many capabilities that were identified under titles 2.1.1, 2.1.2, and 2.1.3 were also gathered under 2.1.2.6; however, other capabilities were identified that addressed the end-to-end case directly and were added to the levels below 2.1.2.6, including the minimum delay capability, ad hoc protocols, seamless interoperation, telemedicine support, etc.

\subsubsection{Space Backbone and Access}

- EML1 \& Lunar Microwave \& Optical Backbone Comm.

- Mars \& Deep Space Microwave \& Optical Comm.

- Earth Comm. Networks

- High Rate Spacecraft Bus \& Networking

- Space Internetworking

- Science Spacecraft O n-Board Data Processing

- Ground Communication Capability

- Architecture Design, Simulation, and Emulation

- Mars Direct to Earth Comm.

\subsubsection{Planetary Networks}

- Mars O rbit to Surface Networks

- Fixed Surface Wireless Local A rea Networks

- Mobile Surface Wireless Local Area Networks

- Planetary Network A rchitecture Design \& Modeling

\subsubsection{Security}

- Secure Space Backbone \& Access Link Communications Architectures and Protocols

- Wireless LAN Standards, Protocols, Secure Networking

- Encryption \& Decryption Components \& Software

- Ground Station Secure Communications

\subsubsection{Navigation}

- Microwave \& Optical Relative Inter-spacecraft Position Measurement and Control Subsystems

- A.utonomous Absolute Navigation References (Lunar/Mars GPS) and Control

- Precision Timing Components and Subsystems

- Absolute Navigation and Relative Position Standards

FIGURE 3. CBS Datasheets Main Points.

\section{CAPABILITY AND TECHNOLOGY DATA GATHERING}

A sample of the CBS worksheet (TABLE 1) shows one representative capability at level 4 with two level 5 subcapabilities below. Left to right by column, the CBS worksheet includes: the capability's breakdown number, its name, and description; the identification (ID) numbers of technologies that address it ("bubble" indicates that the technologies in all the sub-capabilities bubble-up into higher level capability), the units of measurement, and the present-day state of art (SOA) of technologies that can provide the capability; and the H\&REx mission level requirement that the capability addresses, the minimum acceptable value of the capability, and the date at which the technologies must meet TRL 6 (to support a targeted mission phase). Meeting TRL 6 by 2008 infers that the technology is needed to support CEV assembly in Earth orbit. 
TABLE 1. The Capability Breakdown Structure Excel Worksheet.

\begin{tabular}{|c|c|c|c|c|c|c|c|c|}
\hline CBS\# & $\begin{array}{l}\text { Capabil- } \\
\text { ity }\end{array}$ & Description & $\begin{array}{l}\text { Specific } \\
\text { Tech. \# }\end{array}$ & Units & SOA & Requirement & $\begin{array}{c}\text { Minimum Acceptable } \\
\text { Capability }\end{array}$ & $\begin{array}{l}\text { Year to } \\
\text { TRL } 6\end{array}$ \\
\hline 2.1 .1 .1 & $\begin{array}{l}\text { EML1 and } \\
\text { Lunar } \\
\text { Micro- } \\
\text { wave } \\
\text { Backbone } \\
\text { Communi- } \\
\text { cations }\end{array}$ & $\begin{array}{l}\text { Subsystems for } \\
\text { ground station, } \\
\text { space } \\
\text { communication } \\
\text { relays, and } \\
\text { spacecraft to } \\
\text { provide } \\
\text { microwave } \\
\text { capability at all } \\
\text { NASA } \\
\text { frequencies. }\end{array}$ & Bubble & Gbps & $\begin{array}{l}\text { TDRS } \\
1999 \\
\text { Prospec- } \\
\text { tor: } 300 \\
\text { bps } \\
\text { telemetry }\end{array}$ & $\begin{array}{l}\text { Support the } \\
\text { following } \\
\text { application } \\
\text { data rates: } \\
\square \text { TT\&C: }<100 \\
\text { kbps } \\
\square \text { SDTV } \\
\text { Video: } 6 \text { Mbps } \\
\square \text { HDTV } \\
\text { Video: } 19 \\
\text { Mbps } \\
\square \text { Biomedical } \\
\text { control: } 70 \\
\text { kbps } \\
\square \text { Biomedical } \\
\text { monitoring: } \\
\text { 122 kbps } \\
\text { [SCAWG, } \\
07 / 2004]\end{array}$ & $\begin{array}{l}\text { Support multiple data } \\
\text { streams between the Moon, } \\
\text { EML1 Gateway, and Earth } \\
\text { GEO relays for an } \\
\text { aggregate return data rate of } \\
1.2 \text { Gbps from robotic and } \\
\text { science vehicles and } \\
\text { bidirectional data rate of } 1.2 \\
\text { Gbps each direction in } \\
\text { support of human missions. } \\
\text { Low data rate TT\&C } \\
\text { communication } \\
\text { technologies in the S or X- } \\
\text { band to Earth vicinity or the } \\
\text { Moon are mature and are } \\
\text { considered available for use } \\
\text { in the new H\&REx } \\
\text { missions. }\end{array}$ & 2008 \\
\hline 2.1.1.1.1 & $\begin{array}{l}\text { Lunar } \\
\text { Antennas } \\
\text { and } \\
\text { Pointing, } \\
\text { Acquisi- } \\
\text { tion, \& } \\
\text { Tracking } \\
\text { Subsys- } \\
\text { tems }\end{array}$ & $\begin{array}{l}\text { Physically or } \\
\text { electrically } \\
\text { augment } \\
\text { reception and } \\
\text { transmission } \\
\text { and orient } \\
\text { Lunar } \\
\text { backbone } \\
\text { communication } \\
\text { s signals to } \\
\text { optimize data } \\
\text { throughput. }\end{array}$ & $\begin{array}{c}\text { T17 IA } \\
\square \text { T18 } \\
\text { BN } \\
\square \text { T38 } \\
\text { BN } \\
\square T 47 \\
\text { AN } \\
\square T 48 \\
\text { AN } \\
\square \text { T82 } \\
\text { BN } \\
\text { T86 BN }\end{array}$ & $\begin{array}{l}\text { Deg- } \\
\text { rees, } \\
\text { Dia- } \\
\text { meter } \\
\text { (m) }\end{array}$ & $\begin{array}{l}0.1 \\
\text { degree: } \\
\text { DSN } \\
\text { Beam } \\
\text { Wave- } \\
\text { guide } \\
\text { Antennas, } \\
\text { Cassini: } \\
\text { 4m } \\
\text { diameter }\end{array}$ & $\begin{array}{l}\text { Track multiple } \\
\text { mooncraft } \\
\text { simultaneously } \\
\text { [Lunar } \\
\text { Network IND, } \\
01 / 2004 \text { ] }\end{array}$ & $\begin{array}{l}\text { Microwave comm needs } \\
0.01 \text { (or better) degree } \\
\text { pointing accuracy to } \\
\text { communicate with another } \\
\text { node. } \\
\square \text { Need wider beam for } \\
\text { acquisition, autotrack } \\
\text { receivers for tracking and } \\
\text { beam offset for pointing } \\
\text { microwave beam. }\end{array}$ & 2008 \\
\hline 2.1 .1 .1 .2 & $\begin{array}{l}\text { Lunar } \\
\text { Micro- } \\
\text { wave } \\
\text { Transmit- } \\
\text { ter and } \\
\text { Receiver } \\
\text { Subsys- } \\
\text { tems }\end{array}$ & $\begin{array}{l}\text { Convert data } \\
\text { into signals for } \\
\text { transmission; } \\
\text { Convert signals } \\
\text { into data after } \\
\text { reception. }\end{array}$ & $\begin{array}{c}\text { T14 BN } \\
\square \text { T15 } \\
\text { BN } \\
\square \text { T19 } \\
\text { BN } \\
\square T 20 \\
\text { BN } \\
\square T 21 \\
\text { BN } \\
\square T 22 \\
\text { BN } \\
\square T 23 \\
\text { BN } \\
\square T 64 \text { IS } \\
\text { T65 IS } \\
\text { T66 IS } \\
\text { T67 IS } \\
\square+\mathrm{Bubbl} \\
\mathrm{e}\end{array}$ & Watts & $\begin{array}{l}30 \text { Watts - } \\
\text { French } \\
\text { TWT }\end{array}$ & & $\begin{array}{l}100 \text { W TWT Ka-band } \\
\text { transmitters and } 4 \text { tube } \\
\text { power combiner. }\end{array}$ & 2008 \\
\hline
\end{tabular}

TABLE $2 \mathrm{a}$ and TABLE $2 \mathrm{~b}$ show the left- and right-hand sides respectively of four technology data set samples in the Technology Data Summary worksheet. From the left in TABLE 2a are the technology ID number; the technology class and group, which were used as sorting indexes; the source of the information, which was either "assumed" from interpretations of the H\&REx mission requirements and scenarios or are the initials of the person providing the information; the name of the technology; and the description. The next two columns indicate the linkages back into the CBS worksheet: they capture the breakdown numbers and names of the capabilities that the technology addresses. The crosslinking between the CBS sheet and the technology sheet are indicated with the red 
colored numbers in the 2.1.1.1.2 row of the CBS. Each technology, T064-T067, refers back to the CBS sheet. The Impact Rationale column describes the features that are enabled by adopting the technology. The final four columns in the left half capture the estimated degree of difficulty in achieving a successful technology, the estimated state of the TRL, the date at which the technology must reach TRL 6 to support H\&REx missions, and the estimated cost to reach TRL 6. The right half of the technology data sheet in TABLE $2 \mathrm{~b}$ starts with the technology number followed by the name of the performance specification, the performance numbers for today's SOA in that technical area, the projected value of the needed performance, an estimate of the probability of attaining that performance, and the 


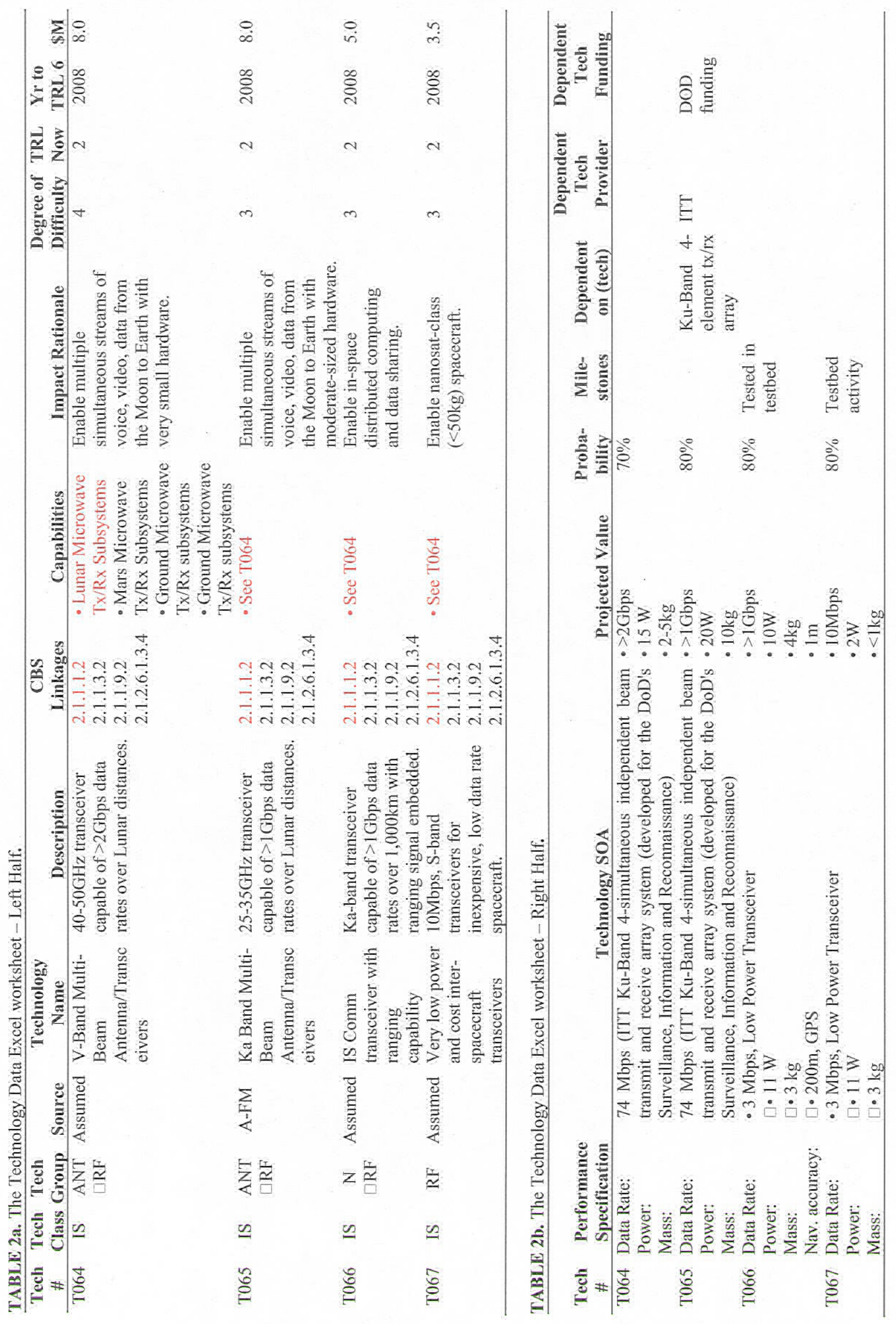


milestone (generally type of test) that must be attained to prove performance is met. The last three columns indicate other technology developments that the success of the line item technology depends upon, the developers of those other technologies, and the state of funding for those technologies. Both of these worksheets reside in the CBS and Technology Excel workbook, along with individual worksheets for each technology data set. There are 224 capabilities identified in the CBS worksheet and 95 technologies.

\section{TECHNOLOGICAL GAPS ANALYSIS}

The most significant technological gaps that were identified in the analyses are shown in TABLE 3 . These items are essentially summaries of multiple capability and technological needs for implementing the H\&REx missions. In each row, the needed capability, current TRL, present SOA, needed performance, and technology development plan for meeting each high level capability are identified. The first item, a space communications relay, is needed to support the assembly of the CEV in Earth orbit and will be needed later to support the CEV in the lunar vicinity as well as the landed human missions. A more capable version of the TDRSS could handle the Earth-orbit CEV missions, while relays in the vicinity of the Moon will be necessary to handle the lunar missions. Microwave, optical, and networking communications system technologies are needed to significantly increase the data rate between Earth and Mars before humans can be sent there. Agile, multipoint, microwave technologies are needed to support communications between the CEV and other in-space vehicles, such as assemblies to become part of the $\mathrm{CEV}$, and to support high speed, bidirectional communications between Moon orbiting relays and the lunar surface. Data networking over high speed links is needed to provide reliable, secure, bidirectional, voice, video, science, engineering, health, and command data with quality of service options and the ability to access the network on demand to enable autonomous operation of the communication systems. Navigational capability should be integrated with communications, that is, incorporated into communication relays and built into human and robotic spacecraft, to enable those spacecraft to "fly" autonomously to locations in the solar system. Wireless communications networks for the surface of Moon and Mars will be necessary to support bidirectional communications between humans, robots, and habitats.

TABLE 3. Main Technological Gaps.

\begin{tabular}{|c|c|c|c|c|}
\hline Needed Capability & TRL & SOA & Needed Performance & Development Plan \\
\hline $\begin{array}{l}\text { Space Relay for CEV } \\
\text { mission LEO to EML1 by } \\
2008\end{array}$ & 4 & $\begin{array}{l}0.15 \text { Gbps (TDRS), } \\
\text { Bent-pipe - no circuit } \\
\text { or packet switching }\end{array}$ & $\begin{array}{l}>1 \text { Gbps Earth relays to CEV, to } \\
\text { Lunar relays and to Lunar } \\
\text { surface; }>100 \text { Mbps Mars, } 10 \\
\text { Mbps deep space }\end{array}$ & $\begin{array}{l}\text { Deploy packet/circuit switched or TDRS } \\
\text { Continuation bent pipe relay, building on relay } \\
\text { technologies from DOD programs, NRO Relay } \\
\text { project. }\end{array}$ \\
\hline $\begin{array}{l}\text { crowave, Optical, } \\
\text { vorking } \\
\text { hications Systems }\end{array}$ & 3 & $\begin{array}{l}300 \mathrm{k} \\
\text { Proxi }\end{array}$ & $\begin{array}{l}\text { d relay, }>100 \\
\text { nd IP interfaces to }\end{array}$ & $\begin{array}{l}\text { op optical and microwave components and } \\
\text { y for long-range communications and } \\
\text { y IP control modules for autonomous demand } \\
\text { inter-nodal links. }\end{array}$ \\
\hline $\begin{array}{l}\text { Agile, Multipoint } \\
\text { Microwave Access Links }\end{array}$ & $3-8$ & $10 \mathrm{Mbps}$ from $\mathrm{S} / \mathrm{C}$ & $\begin{array}{l}\text { form, } 40 \\
\text { C }\end{array}$ & $\begin{array}{l}\text { teered antennas, high speed } \\
\text { trol }\end{array}$ \\
\hline $\begin{array}{l}\text { Reliable, Secure, Bi- } \\
\text { direetional, Voice, Video, } \\
\text { Data Networking Over } \\
\text { High Speed Links }\end{array}$ & $2-3$ & None & $\begin{array}{l}\text { edia access } \\
\text { node } \\
\text { able } \\
\text { ideo, data, }\end{array}$ & $\begin{array}{l}\text { IP compliant } \\
\text { mechanisms in } \\
\text { us demand access, } \\
\text { with legacy systems, } \\
\text { ce IP network. }\end{array}$ \\
\hline $\begin{array}{l}\text { gation and } \\
\text { is for } \\
\text { perations }\end{array}$ & 2 & $\begin{array}{l}\text { Pent tech. } \\
\text { control, } \\
\text { Present USO }\end{array}$ & $\begin{array}{l}\text { Low cost/mass precision timing } \\
\text { onboard spacecraft and use of } \\
\text { external navigational references. } \\
10 \mathrm{~cm} \text { accuracy between } \mathrm{S} / \mathrm{C} ; 10 \\
\mathrm{Mbps} \text { over } 10 \mathrm{~km}\end{array}$ & $\begin{array}{l}\text { GPS near Earth, develop low } \\
\text { oscillator, implement "lightho } \\
\text { relays and ground stations, im } \\
\text { over IP. Develop and integrat } \\
\text { features into access links. }\end{array}$ \\
\hline $\begin{array}{l}\text { Surface Wireless Com- } \\
\text { munications Networks for } \\
\text { Moon and Mars }\end{array}$ & $3-4$ & $\begin{array}{l}802.11 \mathrm{a}, \mathrm{g} \text { (on Earth), } \\
54 \mathrm{Mbps}(30 \mathrm{~m}) \\
802.16,70 \mathrm{Mbps} \\
(50 \mathrm{~km})\end{array}$ & $\begin{array}{l}2014: 802.11 \mathrm{n} \text { on Moon, ranging, } \\
\text { directional antennas, wearable } \\
\text { network transceivers, access } \\
\text { point towers }>100 \mathrm{Mbps}(100 \mathrm{~m}) \text {, } \\
>200 \mathrm{Mbps}(50 \mathrm{~km})\end{array}$ & $\begin{array}{l}\text { Configure wearable wireless systems and access } \\
\text { point towers from commercial units. Perform } \\
\text { extensive field tests while implementing spiral } \\
\text { updates to system and component design to meet } \\
\text { requirements. }\end{array}$ \\
\hline
\end{tabular}

\section{TECHNOLOGY ROAD MAPS}

The development roadmaps for the communications, networking, and in-space computing technologies that will enable the future H\&REx missions technologies are shown in FIGUREs 4-6. The top two rows in each roadmap show the timeline for the missions that include the early CEV missions in Earth orbit, the lunar excursion with the 
CEV, the human landings on the Moon, and the missions to Mars. The roadmap shown in FIGURE 4 indicates when technology developments are needed to enable the future communications and networking infrastructure. This roadmap shows the needed technologies for four types of networks: 1) the very high data rate, long distance, space backbone networks (SBN); 2) the shorter range space wide area network (SWAN) for spacecraft to CEV and relay to lunar or Mars surface communication; 3) surface wireless local area networks (SWLAN) needed for astronauts and robots to work on the surface; and 4) vehicle local area networks (VLAN) that handle inter-subsystem communications on board a spacecraft or vehicle. The triangles and diamonds on the schedule bars show the technology need dates. The three main need dates are the dates the technology must reach TRL 6 , depending on which mission segment the technology must support: 2008 (CEV to low Earth orbit), 2014 (lunar landing), and 2023 (Mars landing). It is expected that the technologies will be continuously improved with the iterations of spiral development.

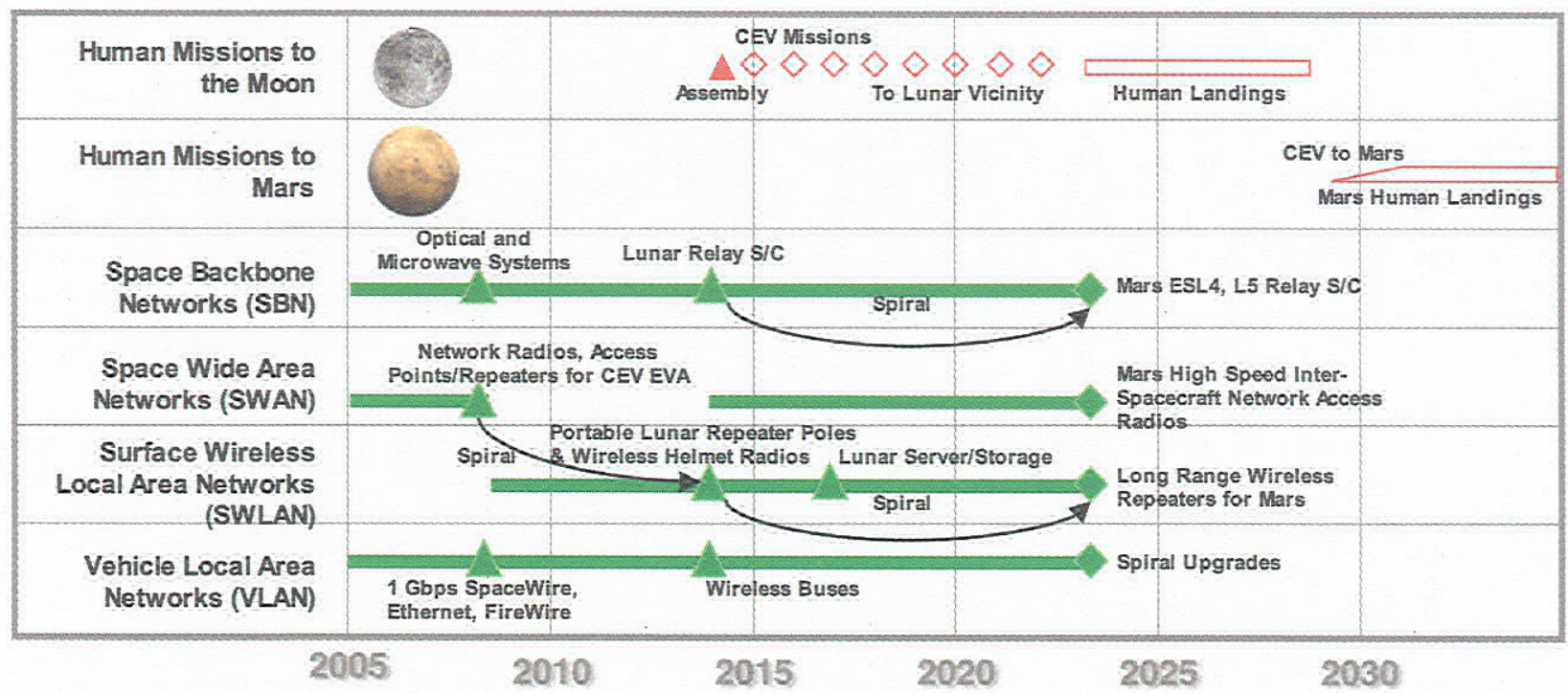

FIGURE 4. The Development Roadmap for Communications and Network Infrastructure Shows Enabling Technologies Needed at TRL 6 by 2008 in Support Of CEV Assembly to Those Needed by 2023 in Support of the Humans to Mars Missions. Software Improvement to Autonomous Communications Will Likely Continue Through the Mars Missions.

The FIGURE 5 roadmap has the timelines for the technologies needed to support end-to-end communications and networking. These technologies are grouped into four areas:

- Autonomous communication technologies support seamless multipoint interoperation, ad hoc network access, and operations in high delay environments;

- Reliable, integrated audio, video, and data technologies support health monitoring, remote inspection, and high rate data and video uplink

- Integrated communications, computing, and data storage technologies support rapid data and software uploads, high definition video, and on-board data processing;

- Secure communications technologies, such as secure protocols and encryption/decryption software, support space-to-ground and ground station security.

The timelines for the in-space computer technologies needed to support many of the networking controller and server functions are shown in FIGURE 6. There are three areas of technology development for space-borne computing: the timeline for innovative architectures and runtime systems technologies shows a processor-inmemory (PIM) computer architecture, its ancillary Field Programmable Gate Array (FPGA) emulation for validating the instruction set and structure, and associated software development of the operating system and the execution environment; the timeline for programming tools and performance optimization includes the development of the programming language, process representation, system libraries, and integrated program support environment; and the timeline for system characterization and modeling includes evaluation of key applications on the PIM-based computer for performance prediction and future impact assessments. These technologies are planned to be ready to support the initial low Earth orbit CEV missions. 


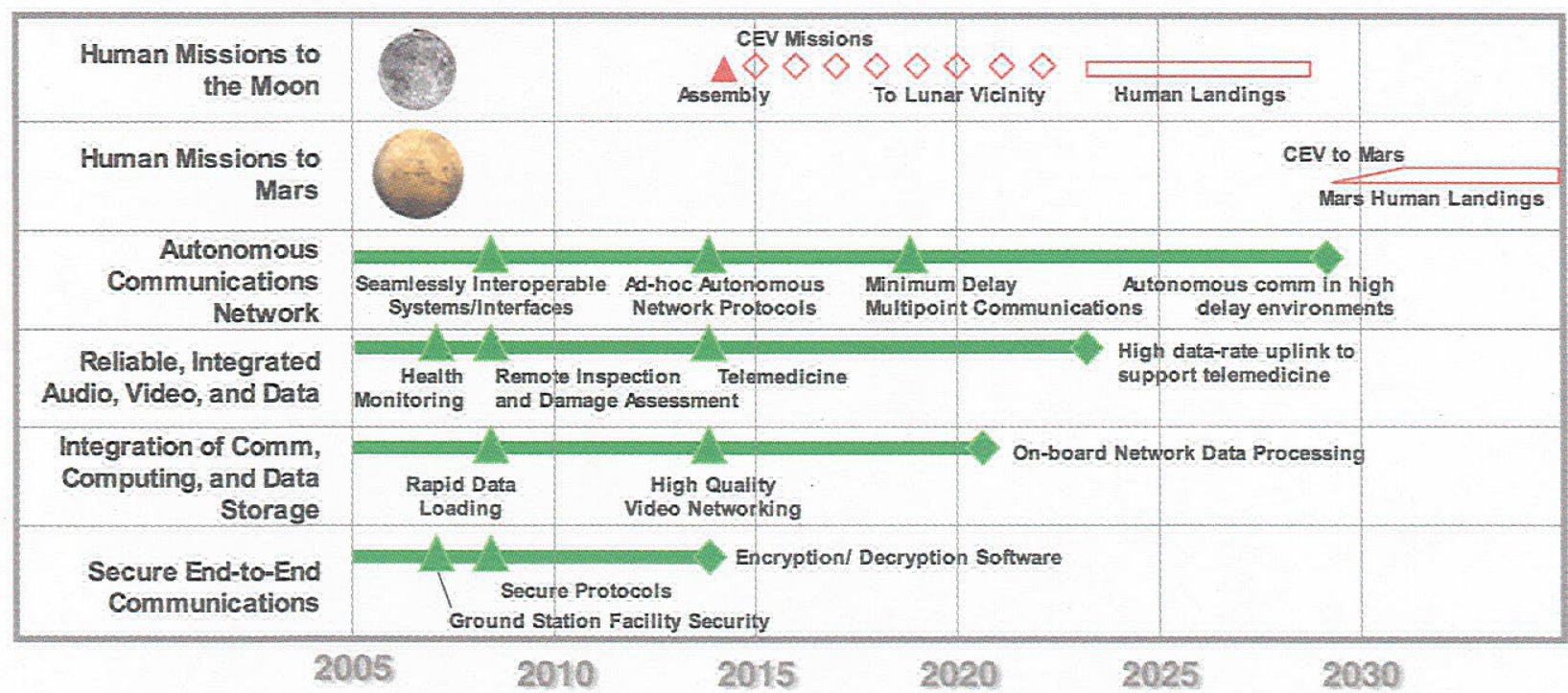

FIGURE 5. The Development Roadmap for End-To-End Communications and Network Enabling Technologies Extends From Obtaining TRL 6 by 2008 in Support of CEV Assembly in Space to Reaching TRL 6 by 2023 in Support of the Humans to Mars Missions. Software Improvement to Autonomous Communications Will Likely Continue Through the Mars Missions.

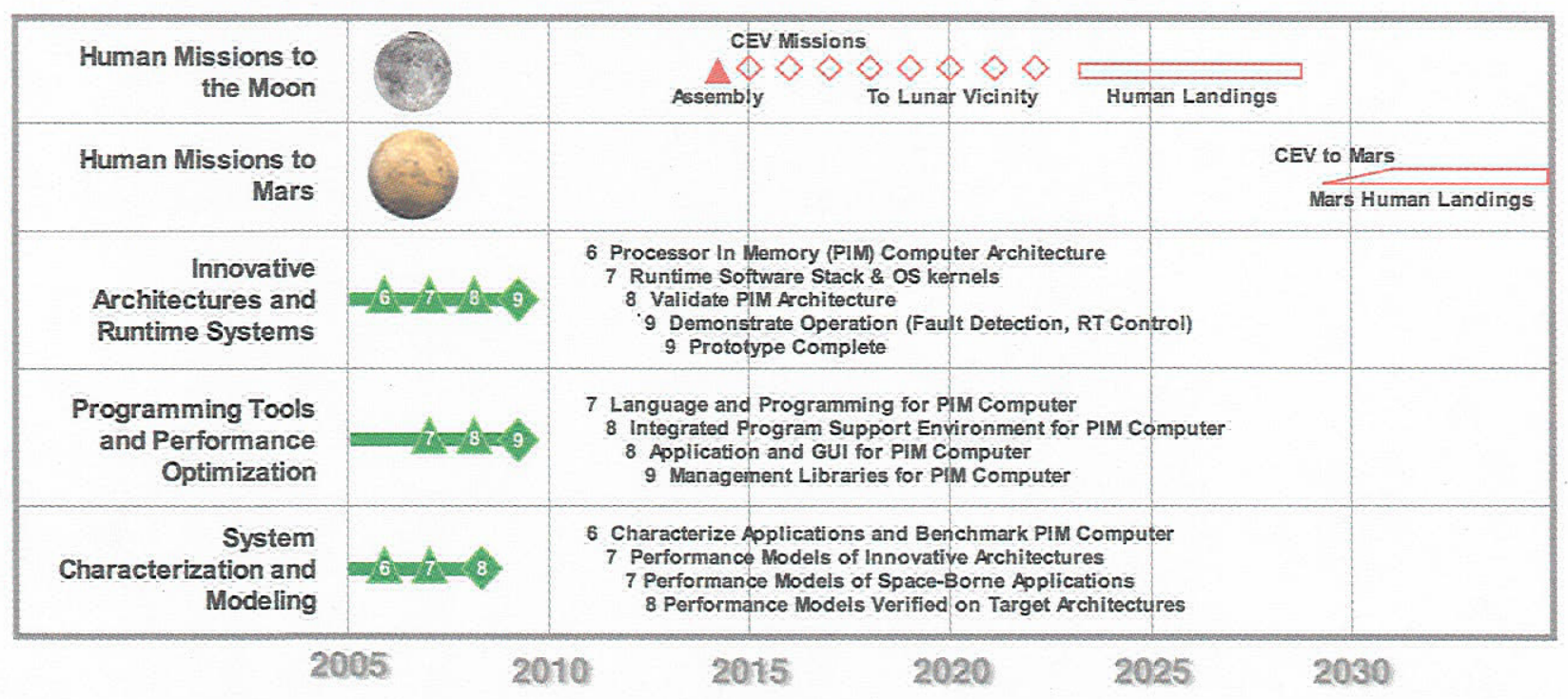

FIGURE 6. The Development Roadmap for In-Space Computer Enabling Technologies to Obtain TRL 6 by 2008 in Support of CEV Assembly in Space.

\section{CONCLUSIONS}

This paper has described the process for determining the technological gaps in communications, networking and processing for providing the capabilities needed to support future space exploration missions. Requirements, design reference missions and experts were utilized in gathering inputs to the process from an architectural view, the mission view and the system view. The mission timeline, outlined in the exploration mission definition, was used to determine the technology development timelines used in the roadmaps. Because the capability and technology data sets represented three viewpoints, they were very large and quite unwieldy to work with. However the process of evaluation from the three views was essential in determining with certainty what technologies were needed and what performance capabilities were needed from the technologies. The Moon is to be the practice ground for human and robotic missions to Mars. Early lunar landing missions are likely to be made on the near side with 4 to 6 astronauts 
and with close monitoring by Earth-based mission operations. The process has enabled the identification of technologies and performances needed to support the communication relays and space-to-space links needed in the early missions. Later missions will land on a lunar pole or the far side and will simulate the independence from Earth-based mission operations that will occur with breaks in line-of-sight communications (as may be expected in Mars missions). The long Mars communication delay times will also be simulated. Astronauts on these missions will be self-reliant and will perform EVAs as a normal life function-as if they were going out for regular walks to work, explore, or just enjoy the countryside. The wireless local area network systems and technologies identified for development through our process will provide the astronauts with the sense of freedom to carry on a conversation and visually share discoveries, collaborate with each other, or handle local emergencies with the assistance of internetworked, helmet-mounted cameras and projectors.

\section{ACKNOWLEDGMENTS}

The authors would like to thank Rupak Biswas of Ames Research Center for his assistance in editing and clarifying the paper; Shirley Tseng of Infinite Global Infrastructures, LLC, and Tom Linsky of the NASA Glenn Research Center for their assistance in coordinating the accumulation of the large amounts of data from the experts and entering that data into the workbook; and Paulette Ziegfield of RSIS at the NASA Glenn Research Center for her extensive edits of the paper.

\section{REFERENCES}

Bhasin, K., and Hayden, J. L., "Developing Architectures and Technologies for an Evolvable NASA Space Communication Infrastructure," 22nd AIAA International Communications Satellite Systems Conference, 2004b.

Bhasin, K., and Hayden, J. L., "Evolutionary Space Communications Architectures for Human/Robotic Exploration and Science Missions," in proceedings of Space Technology and Applications International Forum (STAIF 2004), edited by M. El-Genk, AIP Conference proceedings 699, Melville, New York, 2004a.

Bhasin, K., and Hayden, J. L., "Space Internet Architectures and Technologies for NASA Enterprises," Int. J. Satell. Commun. $2002 ; 20,311-332$.

NASA, First Joint Space Internet Workshop (SIW I), (2000), http://siw.gsfc.nasa.gov/archive/2000-siw/index.html, accessed September 27, 2004.

NASA, Fourth Space Internet Workshop (SIW IV), (2004c), http://siw.gsfc.nasa.gov/, accessed September $27,2004$.

NASA, Human and Robotic Technology Formulation Plan, version 5.1, 2004b, http://exploration.nasa.gov/acquisition.html, accessed October 4, 2004.

NASA: Mars Reference Mission Version 1.0 (1997 - 1999), http://www.swri.edu/swim/human2mars.htm, accessed October 17, 2004.

NASA, Second Space Internet Workshop (SIW II), (2002), http://siw.gsfc.nasa.gov/archive/2002-siw/index.html, accessed September 27, 2004.

NASA, Technology Readiness Levels, version 5, 1999, http://isd.gsfc.nasa.gov/Technology/TRL/TRL.htm, accessed October 1, 2004.

NASA, "The Vision for Space Exploration," 2004a, http://www.nasa.gov/missions/solarsystem/explore main.html, accessed September 26, 2004.

NASA, Third Space Internet Workshop (SIW III), (2003), http:/scp.grc.nasa.gov/siw/, accessed September 27, 2004.

Troutman, P.A., et. al., "Orbital Aggregation and Space Infrastructure Systems (OASIS)", Conference Paper, 53rd International Astronautical Congress; 2002 World Space Congress, 2002. 